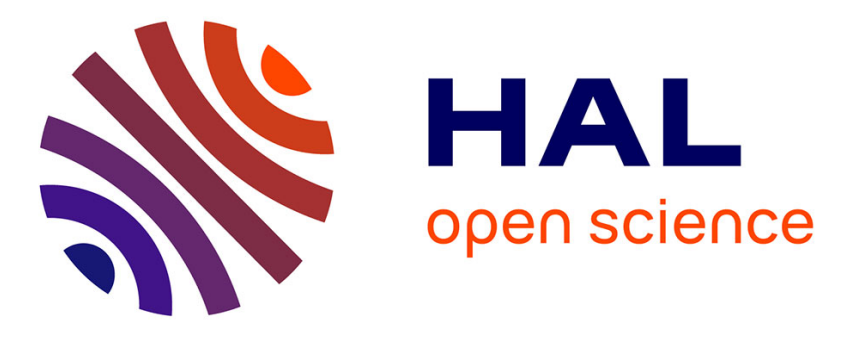

\title{
Easy water stress detection system for vineyard irrigation management
}

Guilhem Brunel, L. Pichon, J. Taylor, Bruno Tisseyre

\section{To cite this version:}

Guilhem Brunel, L. Pichon, J. Taylor, Bruno Tisseyre. Easy water stress detection system for vineyard irrigation management. 12th European Conference on Precision Agriculture, ECPA 2019, Jul 2019, Montpellier, France. pp.935-942, 10.3920/978-90-8686-888-9_115 . hal-02609771

\section{HAL Id: hal-02609771 \\ https://hal.inrae.fr/hal-02609771}

Submitted on 16 May 2020

HAL is a multi-disciplinary open access archive for the deposit and dissemination of scientific research documents, whether they are published or not. The documents may come from teaching and research institutions in France or abroad, or from public or private research centers.
L'archive ouverte pluridisciplinaire HAL, est destinée au dépôt et à la diffusion de documents scientifiques de niveau recherche, publiés ou non, émanant des établissements d'enseignement et de recherche français ou étrangers, des laboratoires publics ou privés. 


\title{
Easy water stress detection system for vineyard irrigation management
}

\author{
G. Brunel ${ }^{1}$, L. Pichon ${ }^{1}$, J. Taylor $^{1}$ and B. Tisseyre ${ }^{1}$ \\ ${ }^{I}$ ITAP, Irstea, Montpellier SupAgro, Univ. of Montpellier, Montpellier, France \\ guilhem.brunel@supagro.fr
}

\begin{abstract}
The monitoring and management of vine water stress is a major issue for many vineyards. A method based on the observation of shoot tip growth makes it possible to determine the water stress of plants without the drawbacks of standard instrumental methods. However, this "shoot tip" method is rarely used in the field, as observations and index calculations need to be done manually. Therefore, while the data collection can be relatively quick, data interpretation is not well supported. This article presents a mobile application, 'Apex', developed to answer this challenge. The Apex application allows a simple and fast recording of observations and a pooling of spatialized observations. The developed application makes it possible to envisage the collaborative water stress monitoring with crowdsourcing observations.
\end{abstract}

Keywords: vine water status, shoot tip index, smartphone application, Vitis vinifera.

\section{Introduction}

Many authors have demonstrated the significant role of water status in vine behaviour and berry composition (Cifre et al. 2005). Monitoring vine water status is of importance for yield and quality management purposes. The vine water status naturally changes over time according to the climatic characteristics of the season. In addition, many authors have shown that it presents a significant spatial variability at different scales: at the within field level (Tisseyre et al. 2005), at the vineyard level (Taylor et al. 2011) and at the regional scale (Baralon et al. 2012). Characterizing the temporal and the spatial variability of vine water status is therefore of paramount importance at these different scales to provide the wine industry with relevant decision support tools: either for irrigation scheduling or for other management practices in the case of non-irrigated vineyards.

Several standard instrumental methods have been proposed to measure plant water status (Acevedo-Opazo et al. 2008). However, these are not easy to obtain, since they are manual techniques requiring heavy materials and specific skills. These constraints make systematic spatio-temporal vine water status measurements difficult to carry out and time-consuming. In order to overcome these constraints, simple alternative approaches have been proposed (Acevedo-Opazo et al. 2008). Among them, the shoot tip index (STI) proposed by Trambouze et al. (2009) and Rodríguez-Lovelle et al. (2009) is of interest as it is based on simple observations of vine shoots. Although more imprecise than standard methods, it has the advantage of being easily achievable without expensive equipment and by inexperienced operators. This method is therefore interesting to monitor temporal and spatial changes in vine water status when several operators may be involved (workers, growers, advisors) in collecting observations.

However, the STI method has some limitations that limits its dissemination: i) it requires the observers to count the Apex (end of shoots) of approximately 50 shoots and 
to class them into three different classes (full growth, moderate growth, growth stopped), ii) the STI needs to be calculated based on the Apex observations and then iii) the observed STI value needs to be recorded and transferred to a database with additional information, like the time and the location of observation. Although simple, these different operations can be cumbersome because they require information to be recorded and a calculation to be made in the field. The aim of this article is to present a mobile application, called ApeX, whose purpose is to tackle this issue by providing a simple tool for: i) collecting Apex notations, ii) calculating STI, iii) geo-referencing and timing STI observations and iv) sending STI observation to a server.

\section{Materials and methods}

The specifications of ApeX were defined by involving the end-users in development and the developments followed the precepts of UX Design (Hekkert 2011). This step made it possible to highlight the characteristics that defined the technical choices of the application. The main ones are summarized hereafter:

- Dissemination: The application must be widely deployable to different organisations and stakeholders (growers, advisors, etc.) whose smartphone (model, operating system, etc.) may be different,

- Accessible: Users are not necessarily familiar with professional applications. The use must be simple and user-friendly while maintaining the data integrity,

- Offline mode: Network coverage is variable according to the areas of use, the application must operate in offline mode as well as in connected mode.

The developments followed the precepts of UX Design, which refers to a person's emotions and attitudes about using a product or service. User experience is dynamic over time due to changing circumstances of usage and changes to individual systems, as well as the wider usage context. User needs can be described in three ways: Why, What and How. "Why" corresponds to the motivations, values and vision of the context. "What" describes the features and functionalities necessary to achieve the "Why". And finally, "How" corresponds to the way of handling and aesthetics. As shown in Figure 1, the application design process follows an iterative cycle consisting of four phases based on Design-thinking methods:

- First phase consists of discovering user needs, requirements and constraints,

- Second phase consists of imagining all the scenarios and solutions to meet the needs. At the end of this phase, all participants make a pragmatic choice on the new features to be added,

- Third phase consists of offering the user a visual set that is both operational and functional. The aim is to combine comfort of use without losing efficiency,

- Fourth phase consists of quickly implementing the chosen characteristics and performing tests in real conditions by the end user.

Unlike a traditional approach where a finalized solution is produced after the third phase, here the cycle will be renewed after a test period. This is needed because the user experience is dynamic and constantly changing over time. Four experts were integrated 
into the ApeX development cycles (6 complete cycles), these users were able to test and validate the various updates.

ApeX was developed as a hybrid app. A hybrid app is one that combines elements of both native and web applications. Hybrid applications execute within wrappers that are targeted to each platform, and rely on standards-compliant application programming interface (API) bindings to access each device's capabilities, such as embedded sensors, data, network status, like a native application. A hybrid app approach was chosen to facilitate the dissemination of the application across more than one platform, without having to re-implement it with each platform's language and tool set.

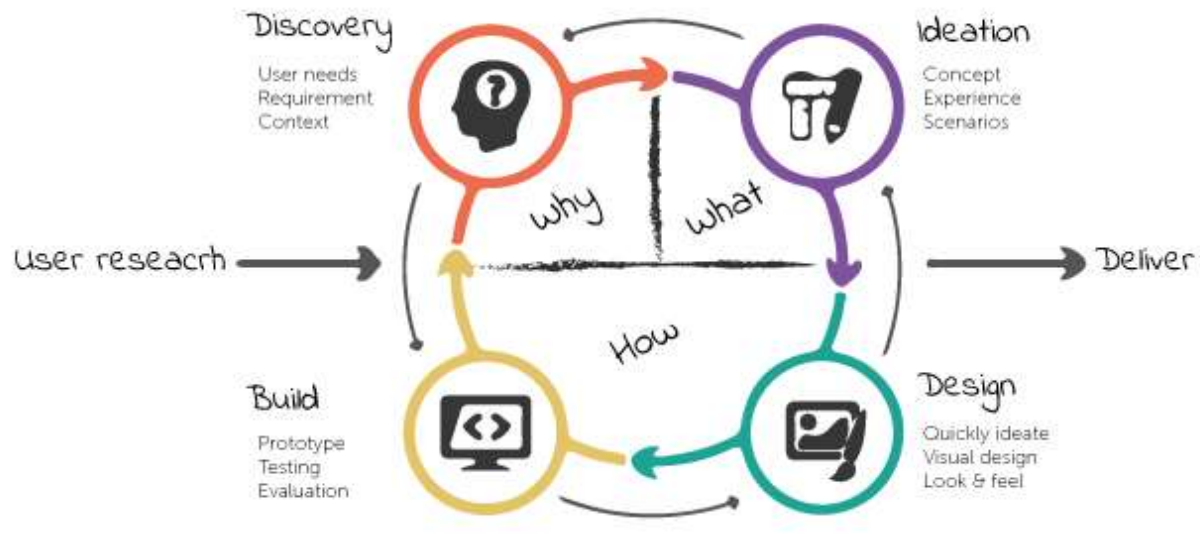

Figure 1. This diagram shows the iterative design process to discover, define, design and develop a digital solution that meets user needs

The development of the application was programmed using Ionic framework based on Apache Cordova (Griffith 2017). Apache is an open-source mobile development framework, which allows the use of standard web technologies - HTML5, CSS3, and JavaScript - for cross-platform development. AngularJS is a JavaScript-based opensource front-end web application framework to address many of the challenges encountered in developing applications. The JavaScript components complement Apache Cordova. It aims to simplify both the development and the testing of such applications by providing a framework for client-side model-view-controller (MVC) and model-view-view model (MVVM) architectures, along with components commonly used in rich internet applications. The application uses Typescript, a superset of JavaScript, which allows access to a number of extra features, such as type of declarations and interfaces. JavaScript code runs on a NodeJS server, which is a platform built on Chrome's V8 JavaScript engine. It differs from other platforms in that it uses a non-blocking approach to perform I/O asynchronously. This workspace is coupled with Gulp, which offers a toolkit for automating time-consuming tasks in the development workflow (Fig. 2).

All data created and inserted into the application is synchronized with the ApeX cloud when the smartphone has a $4 \mathrm{G}$ or Wi-Fi connection. To operate in areas without a network, all data is stored in a local database supported by SQLite and are automatically synchronized on a MySQL server database when the network allows it. Communication between the smartphone and the server is provided by a JavaScript API. The application uses the smartphone's GNSS to locate observations. The web application was designed and developed to quickly and efficiently visualize the collected data. The web 
application was developed using HTML5 mark-up language, CSS3 styling and WordPress API for a responsive web design. It automatically adjusts to different screen sizes, allowing it to be easily viewed on any device or at any screen resolution.

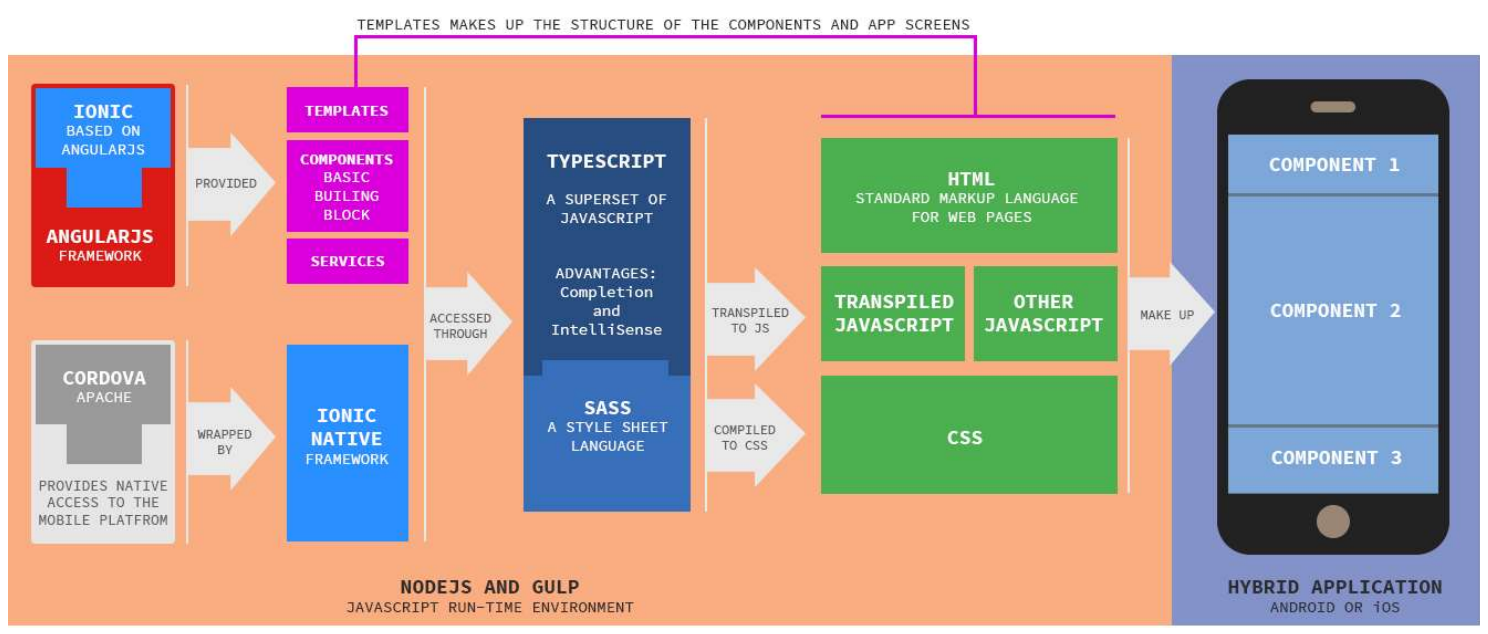

Figure 2. Technologies used for the development of ApeX

The ApeX app was tested by twenty users in the summer of 2018 in the Occitanie and Provence-Alpes-Côte d'Azur regions of southern France. One session represents fifty observations of shoot tips in a vineyard field, which is necessary for the STI to be calculated to estimate vine water stress. More than 1500 sessions distributed over 270 different vine fields were collected over the season.

\section{Results}

Application interface

The calculation page is divided into three areas (Fig. 3a): i) a list to choose the field that the observation is performed in. This list is ordered according to the user's GNSS position, ii) three buttons for shoot tip selection. This area takes up $70 \%$ of the screen for comfort of use. Each button has a different vibration to indicate user choice, iii) a dynamic counter at the bottom of the screen to allow the user to know the number of observations made. A long vibration of the phone indicates the achievement of the fifty observations necessary to calculate the STI. The STI button is only clickable after fifty observations to compute STI.

The home page (Fig. $3 \mathrm{~b}$ ) navigates to the calculation pages and allow visualization of the results. For each field, the user can access i) the current growth rate of the vine (vigour), ii) the growth dynamics based on past and current growing STI rates and iii) a scale range of the current STI (water stress). To improve the quality of the user experience, other interfaces have been developed (instructions, contact form, field characteristics, etc...).

\section{Spatial distribution of observations}

More than 1500 sessions (each of 50 shoot observations), from 22 operators from 7 different companies (extension services, advisers and research institutes) were collected with the ApeX app during 2018. Figure 4 shows the sessions collected at two different scales: the within-field scale (Fig. 4a) and the regional scale (Fig. 4b). 

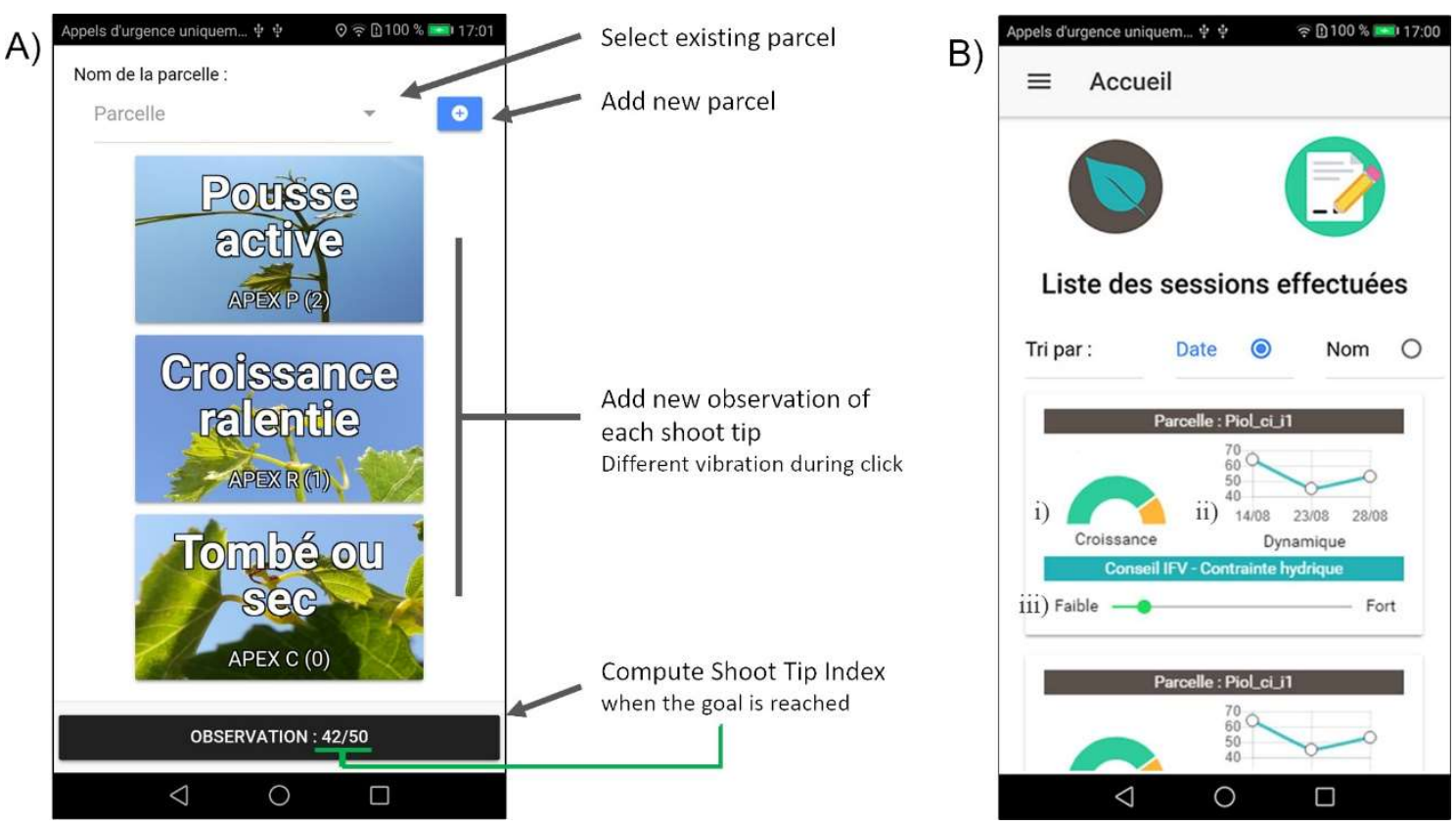

Figure 3. Screenshot of both main interfaces of ApeX

At the within field scale, (Fig. 4a), the 50 observations were plotted to provide more detailed information on the monitoring that has been carried out. Some observations were positioned outside the fields. This result can be explained by the low quality of the smartphone's geolocation sensor but also by poor operating conditions (fast switching on of the smartphone, locations with poor positioning signal quality etc...). All together, these problems resulted in spatial outliers. It highlighted the need to identify and to correct outliers before the app is made available to professionals.

At the regional scale, the data were collected mainly around the Mediterranean basin (Fig. 4b). This spatial distribution of the observations was mostly driven by the location of partners who were interested in using the ApeX application and for whom the monitoring of water stress is a major issue.
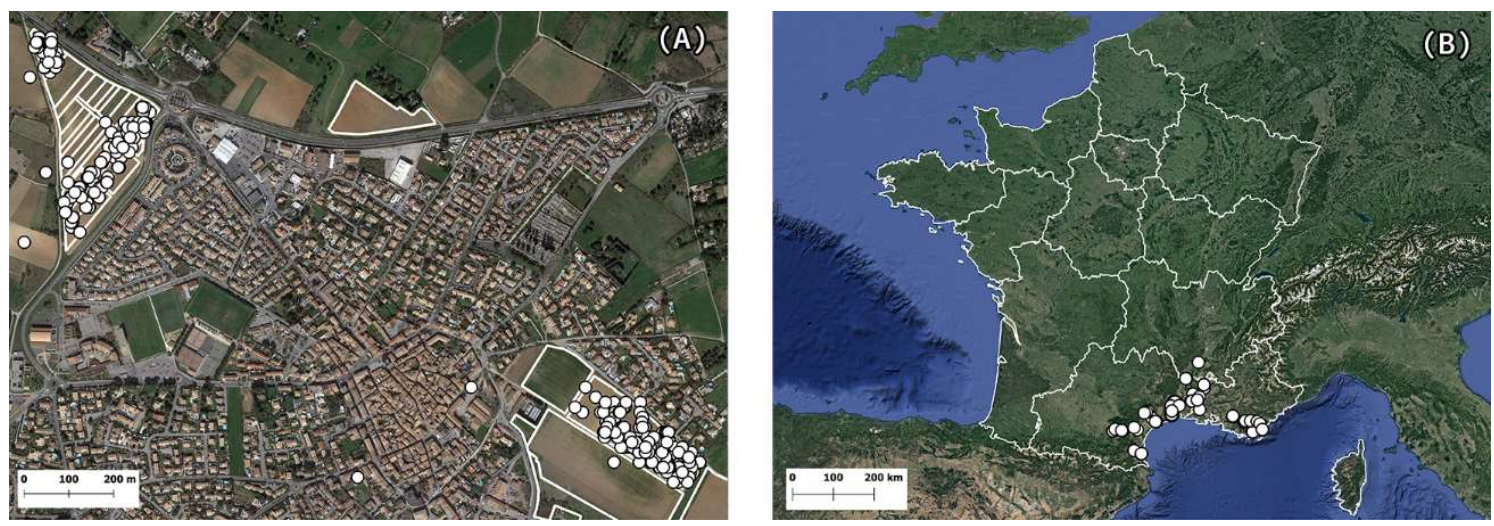

Figure 4. Observations collected with Apex during 2018 in southern France, a) example at the within-field level, b) spatial distribution of measurements at the regional level. 
Temporal distribution of observations

Figure 5a shows the number of sessions collected during the 2018 season for each twoweek interval. The first sessions were made at the beginning of June with about fifty sessions over the first two weeks. The number of sessions then increased sharply to $\sim 400$ over the same interval at the beginning of July. It then decreased during August, with only a few sessions during September and October. ApeX was predominantly used to decide the date of the first irrigation during the dry summer period when vine water stress reached critical values. In the Mediterranean region, this decision is usually taken in July. The increased and decreased number of observations corresponded respectively to the start and end of a period of risk for the vine. Most users who started using the application kept on using it during the season. This indicated that it met users' expectations and on the other hand that its usability was satisfactory to them.

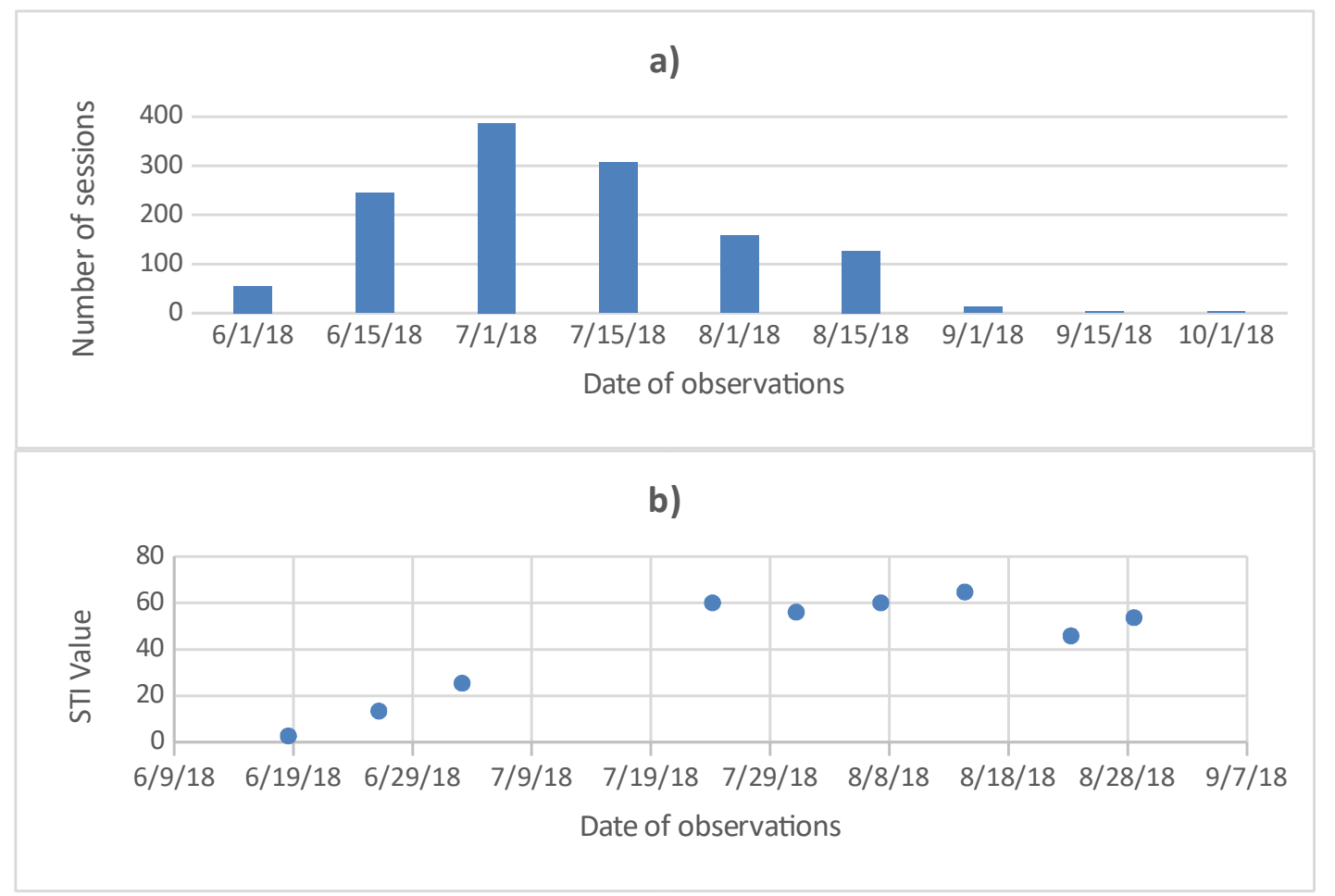

Figure 5. Use of ApeX over the season and examples of results (June - October) (a) the count of sessions made each fortnight and (b) the average recorded STI at approximately weekly intervals in a field.

Figure $5 \mathrm{~b}$ shows an example of the evolution of the STI indicator over the season in a vineyard. The STI was close to 0 on June $19^{\text {th }}$, corresponding to significant vegetative growth and no water restriction. The STI increased steadily during June, as vine growth decreased with the occurrence of a significant water restriction. The STI then stabilized at around 60, indicating moderate to weak growth between mid-July and early September. Slight variations in the STI were observed during this period. This information, combined with agronomic expertise, could be used to identify periods of water stress as a decision support vineyard management. 


\section{Discussion}

The development of ApeX followed the MVC pattern, in which the user interface (presentation), functional process logic (decision rules code), computer data storage and data access were developed and maintained as independent modules. This application architecture provided a flexible model and a reusable application. By segregating an application into tiers, it was possible to refine the existing model or add new ones based on shoot tip analysis, without having to rebuild the entire application.

The specifications of ApeX were realized by involving the end-users and the developments followed the precepts of UX Design. This reduced development times by directly integrating feedback and requests for improvement. A suggestion form was added in the first version so that the user could feedback directly during use. Consequently, some changes were made, for example, vibrations during clicks were added to allow the user to feel actions without having to check the screen at each observation. This basic feature was widely appreciated, as it considerably increased user comfort. Users also felt involved in the application, which explains the success and the large amount of data collected during the first test season.

The results of this study showed that the ApeX application allows the collection of observations on vine growth in a simple and rapid way. These observations were georeferenced and shared at different scales. This work assumed that the ApeX method has been validated by comparison with reference methods in previous work (Trambouze et al. 2009). New experiments will have to be conducted to validate whether the use of the ApeX application improves or alters the relationship with reference measurements. In this work, the use of the application was restricted to a limited number of users, but the number of sessions collected was still relatively large. When the ApeX app is freely released to the wider public, it should collect a very large number of sessions at very different scales. This wider use will be an opportunity to develop a tool to help monitor water stress at a large scale. However, it does raise the question of the quality of the data that will be produced by the application. This aspect is indeed a major challenge in the use of crowdsourced data (Minet et al. 2017). It will be necessary to set up mechanisms to characterize and improve the quality of the data collected. Finally, it seems relevant to study how this new data source, that provides data in large quantities of low to medium quality, can complement and enrich the reference data.

\section{Conclusion}

The ApeX application was developed to monitor vine water status using a simple and robust method that can potentially be used by stakeholders with very different skills. The application is free of charge. It is a novel decision support tool that can be an interesting alternative for small growers who cannot afford alternative commercial services. The version presented in this work is a first approach. However, after a first season of use, the results showed that users have adopted the application throughout the monitoring season. This indicated that it meets a need and that the interface is appropriate.

The app can be used at the field or the within-field scale for temporal or spatio-temporal monitoring of vine water status. It can also be used at a regional scale by aggregating data, although one limitation remains the quality and imprecision of the information obtained with this type of approach. 


\section{Acknowledgements}

We thank the "Institut Français de la Vigne et du Vin" for their involvement throughout the development of the application. We also thank the Chambers of Agriculture who participated in the test campaign during the summer of 2018. The Occitanie region for its financial support in the frame of the crowd-viti project (repere project).

\section{References}

Acevedo-Opazo C., Tisseyre B., Ojeda H., Ortega-Farias S. and Guillaume S., 2008. Is it possible to assess the spatial variability of vine water status? Journal International Des Sciences De La Vigne et Du Vin 42, 203-220.

Baralon K., Payam J-C., Salancon E. and Tisseyre, B., 2012. SPIDER: spatial extrapolation of the vine water status at the whole denomination scale from a reference site. Journal International Des Sciences De La Vigne et Du Vin (3), 167175.

Cifre J., Bota J., Escalona J.M., Medrano H. and Flexas J., 2005. Physiological tools for irrigation scheduling in grapevine (Vitis vinifera L.). An open gate to improve water-use efficiency? Agriculture, Ecosystems \& Environment 106, 159-170.

Griffith C. 2017. Mobile App Development with Ionic Revised Edition Cross Platform Apps with Ionic Angular \& Cordova. Sebastopol, CA, USA: O'Reilly Media.

Hekkert P., van Dijk M. B. 2011. Vision in design: A guidebook for innovators. Amsterdam, The Netherlands: BIS Publishers.

Minet J., Curnel Y., Gobin A., Goffart J.P., Mélard F., Tychon B., et al. 2017. Crowdsourcing for agricultural applications: A review of uses and opportunities for a farm sourcing approach. Computers and Electronics in Agriculture. 142, Part A, $126-138$.

Rodriguez-Lovelle B., Trambouze W., and Jacquet O., 2009. Evaluation de l'état de croissance végétative de la vigne par la «méthode des ApeX» (Evaluation of the vegetative growth status of the vine by the "ApeX method"). Le Progrès agricole et viticole, 126 (4), 77-88.

Taylor J.A., Acevedo-Opazo C., Pellegrino A., Ojeda H. and Tisseyre B., 2011. A comment on inter-field spatial extrapolation of vine (Vitis vinifera L.) water status. Journal International Des Sciences De La Vigne et Du Vin (2), 121-124.

Tisseyre B., Ojeda H., Carillo N., Deis L. and Heywang M., 2005. Precision viticulture and water status, mapping the pre-dawn water potential to define within vineyard zones. In: H. R. Shultz (Ed.). Proceedings of 14th GESCO congress, Geisenheim, Germany: Groupe d'Etudes des systèmes de Conduite de la Vigne, pp 23-27.

Trambouze W., Rodriguez-Lovelle B. and Jacquet O., 2009. The shoot tip method: a practical tool to evaluate the vine growth status. In: Viticulture \& Enology, XVIth international Giesco Symposium. Davis, California, pp 112-120. 